\title{
Team structure, team climate and the quality of care in primary care: an observational study
}

\author{
P Bower, S Campbell, C Bojke, B Sibbald
}

See editorial commentary, p 243

Qual Saf Health Care 2003;12:273-279

See end of article for authors' affiliations

.....................

Correspondence to: Dr P Bower, NPCRDC, 5th Floor, Williamson Building, University of Manchester, Manchester M13 9PL, UK; peter.bower@man.ac.uk

Accepted for publication 18 March 2003
Objectives: To determine whether practice structure (for example, list size, number of staff) predicts team processes and whether practice structure and team process in turn predict team outcomes Design: Observational study using postal questionnaires and medical note audit. Team process was assessed through a measure of "climate" which examines shared perceptions of organisational policies, practices, and procedures.

Setting: Primary care.

Subjects: Members of the primary health care team from 42 practices.

Main outcome measures: Objective measures of quality of chronic disease management, patients' evaluations of practices, teams' self-reported ratings of effectiveness, and innovation.

Results: Team climate was better in singlehanded practices than in partnerships. Practices with longer booking intervals provided superior chronic disease management. Higher team climate scores were associated with superior clinical care in diabetes, more positive patient evaluations of practice and self-reported innovation and effectiveness.

Conclusions: Although the conclusions are preliminary because of the limited sample size, the study suggests that there are important relationships between team structure, process, and outcome that may be of relevance to quality improvement initiatives in primary care. Possible causal mechanisms that might underlie these associations remain to be determined.
$T$ he growth in the number of healthcare professionals working in primary care in the last 20 years has been well documented. ${ }^{1}$ The potential advantages of working in integrated teams in primary care are threefold and involve increases in (1) task effectiveness (improving patient health and satisfaction with care); (2) mental health (the morale and well being of team members); and (3) team viability (the degree to which a team will function over time). ${ }^{23}$ However, there has also been a realisation that the structural changes in healthcare teams may not have led to the expected improved outcomes.

Structural changes may only be translated into positive outcomes if processes at the level of the team are effective. Obstacles to the smooth function of primary healthcare teams include interpersonal and professional issues such as role conflicts, professional boundary disputes, value differences, and tensions concerning power, autonomy and control. ${ }^{4-7}$ Increasing the number and range of staff may mean that staff have more support available (which may increase morale) and that patients have access to a wider range of clinical skills (which may improve health outcomes). However, these benefits may not be realised if processes among the team are an obstacle-for example, staff support and effective sharing of clinical tasks may be hampered by professional role conflicts or poor communication.

Concepts that may be of relevance to team processes are "culture" and "climate". Although they are not identical concepts, both are concerned with psychosocial processes at the level of the group rather than the individual. Climate represents a team's shared perceptions of organisational policies, practices and procedures, ${ }^{8}$ and is proposed to comprise four broad factors:

(1) Shared vision and objectives, "an idea of a valued outcome which represents a higher order goal and a motivating force at work".
(2) Participative safety, defined as a situation in which involvement in decision making is motivated and occurs in a non-threatening environment.

(3) Commitment to excellence, involving a shared concern with quality of task performance.

(4) Support for innovation, the support of attempts to introduce new ways of working.

Team climate is viewed as a variable possessed by an organisation that can be described, measured, and manipulated to enhance the effectiveness of the organisation. ${ }^{9}$ In line with this approach, a questionnaire to measure climate (the Team Climate Inventory; TCI) has been developed and received preliminary validation in primary care teams. ${ }^{8}$ The idea that climate is "shared" can be examined using statistical procedures to determine agreement and consensus. ${ }^{10}$

The measurement of team outcomes can be problematic. In some teams such as those in business or the airline industry there may be an obvious high priority outcome (financial performance, low error rate). However, in health care the issue is more complicated in that there are numerous views as to the goals of the team, such as those highlighted by policy documents, the needs of patients, and the views of the healthcare professionals themselves. A "constituency approach" has been used, using multiple stakeholders (patients, professionals, and other groups) to define outcome criteria. ${ }^{11}$ The criteria included responsiveness to patients, quality of care, staff development, and organisational development.

Poulton and West examined the relationship between team structure (for example, practice size), process (that is, climate), and outcomes. ${ }^{12}$ GPs, nurses, and administrators from 46 primary care teams completed the TCI and also provided ratings of the following outcomes: teamworking, quality of professional practice, patient centred care, and overall effectiveness. The climate factor "shared objectives" was most highly related to team effectiveness. Team processes explained more outcome variance than practice structure. 


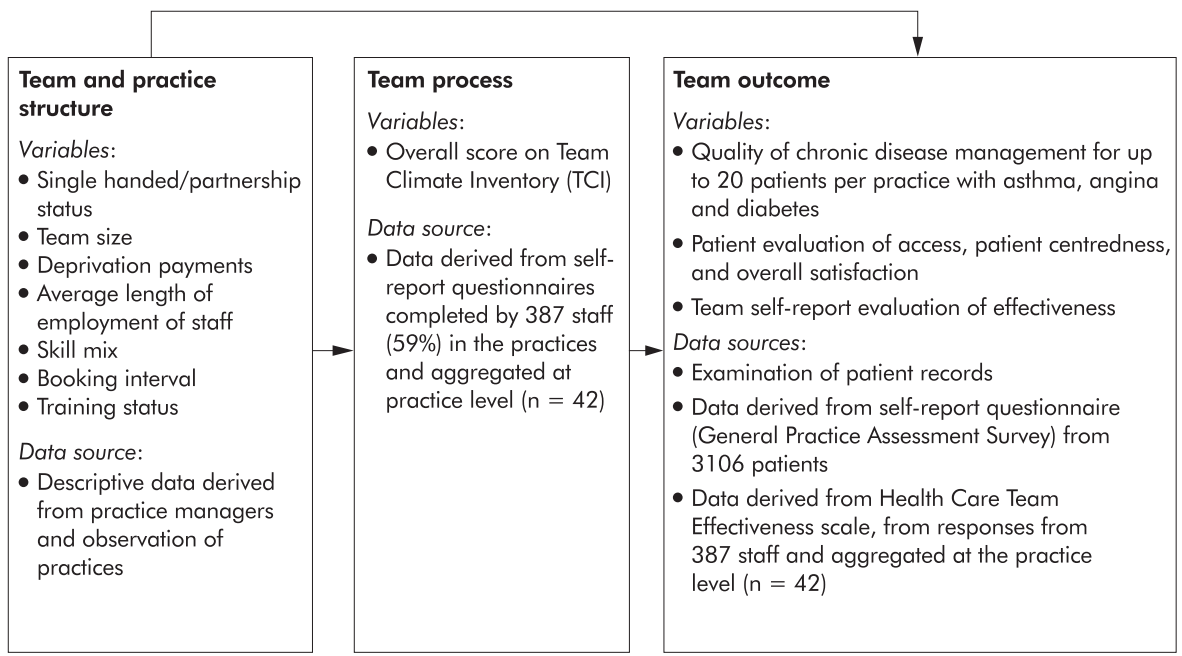

Figure 1 General model of structure-process-outcome relationships and details of the exact variables used in the analysis.

Two studies from Spain have also examined the relationship between teamworking and effectiveness in primary health care teams. ${ }^{13}{ }^{14}$ Both found that aspects of teamworking were related to outcomes such as job satisfaction, efficacy, and quality as rated by users of the service.

The major limitation of these studies was that most outcome measures used staff self-reports and only one study extended this to include objective criteria and patient evaluations. ${ }^{13}$ Measures of effectiveness would have greater credibility if based on external measurement using objective criteria. Team climate has been found to predict objective measures such as sickness absence in doctors, ${ }^{15}$ but this study was not performed in primary care.

Another problem in the UK study ${ }^{12}$ was that the teams were nominated by local organising teams involved in facilitating training workshops. The teams were thus actively committed to teamwork, which may threaten external validity.

The present study sought to replicate this previous work but to overcome these internal and external validity problems through the use of externally measured outcomes and an attempt to recruit a representative sample of primary care teams. Two questions were studied:

- Does practice structure (for example, list size, number of staff) influence team processes (that is, climate)?

- Do practice structure and team process in turn predict objectively measured team outcomes?

The general model of the relationships is shown in fig 1.

\section{METHODS}

This study (conducted in 1998-9) was based on a quality assessment project using previously published quality measures which are detailed below. ${ }^{16}$ The sample consisted of a stratified random sample of 60 English general practices from six health authorities, selected to be nationally representative for rurality and deprivation. Within each authority, 10 practices were selected randomly to be representative of their health authority for practice size, training status, and deprivation payments. Where a practice refused participation, another with similar characteristics was selected and invited to participate; 60 out of 75 practices approached (80\%) agreed. Practice staff were requested to complete measures of team climate and effectiveness. The measures used in the study are shown in fig 1 .

\section{Measures of structure}

The structural variables were singlehanded status (binary variable for singlehanded or partnership); team size (number of employed staff); existence of deprivation payments to the practice (binary variable); training status of the practice (binary variable); mean length of employment of staff at the practice; routine booking intervals for patient consultations (categorical variable with three categories for 5, 7.5, and 10 minutes); and variables representing skill mix (see below). Data were collected from practice managers and through observation during visits by researchers to the practices.

Skill mix variables were created based on team composition. At present there is no clear definition of skill mix which can refer to the mix of skills, grades, and disciplines within a team. Because no data were available on individual skills, the focus in the current study was on disciplinary mix. There are no validated measures of disciplinary skill mix available, so three exploratory measures were calculated to examine the concept:

- the ratio of doctors to nurses (SMl);

- the ratio of doctors to non-medical clinical staff (SM2);

- the ratio of clinical to administrative staff (SM3).

\section{Measures of process (team climate)}

The TCI is a 65 item measure with six subscales rated on 5 point scales (from "strongly agree" to "strongly disagree"):

- participation (the "safety" of the decision making environment): items concern issues such as sharing information, influence of staff on each other, feelings of being understood and accepted;

- support for innovation (team support for new ideas): items concern issues such as openness to new ideas and sharing resources:

- reflexivity (team discussion and review of procedures): items concern issues such as review of objectives, communications, and decisions;

- task orientation (team emphasis on monitoring quality): items concern issues such as monitoring each others' work, appraisal of weaknesses, and provision of practical ideas and help;

- clarity of objectives (team understanding of objectives): items concern issues such as agreement about objectives and their perceived usefulness;

- teamworking (degree to which teamworking is valued): items concern issues such as interdependence and perceived liking for teamworking.

The latest version of the questionnaire is based on earlier versions which have demonstrated construct, predictive, and discriminant validity. ${ }^{8}$ In order to avoid excessive hypothesis 
testing, an overall score on the TCI was computed based on the summed subscales; this score ranged from 6 to 30 .

\section{Quantification of team climate}

Team climate measures are applied at an individual level, yet the definition of climate requires that the perceptions are shared. Thus, a measure of consensus is required to provide a justification for the aggregation of individual scores and evidence for the construct validity of the team level means. ${ }^{17}$ The scores of individual members were aggregated to provide an overall team climate score based on the mean of the individual team members. The $r_{\mathrm{wg}(j)}$ measure of agreement was used, ${ }^{18} 19$ which is an index of agreement among judges concerning ratings of single items or homogenous scales. Although the use of the index has been criticised, ${ }^{20}$ it was used in the present study to ensure comparability with previous analyses of the TCI. Scores of above 0.7 demonstrate acceptable agreement among respondents.

\section{Measures of outcome \\ Health Care Team Effectiveness (HCTE) scale}

The HCTE is a self-report measure of team effectiveness with 21 items measured on 7 point scales (from "not at all" to "to a great extent") completed by the health professional. The development of the scale has been described elsewhere, ${ }^{11}{ }^{21}$ although validity is largely restricted to face validity at present. The items are combined into three factorsprofessional practice (audit, setting protocols, use of research evidence); teamworking (professional development, equal opportunities); and patient centred care (information provided to patients, provision of complaints procedure) —and an overall measure of team effectiveness (the mean of all items). One additional factor (perceived team innovation) was measured using five additional items measured on 5 point scales (from "highly stable" to "highly innovative"). Again, to restrict multiple hypothesis testing, only the overall score on the HCTE was used in the analyses (range 1-7), together with the separate measure of innovation (range 1-5).

\section{Chronic disease management}

Up to 20 patients with adult asthma, angina, and type 2 diabetes were selected randomly from disease registers. The mean number per practice was 18 (range 6-20) for angina, 19 (range 13-20) for asthma, and 18 (range 9-20) for diabetes. Data were extracted from records by researchers to identify processes defined by experts as "necessary" to provide high quality care. ${ }^{22}$ An example of the criteria used is shown in box 1. The reliability of data extraction was tested ${ }^{23}$ and only reliable variables were included. Data items for chronic disease management were scored on a $0 / 1$ basis depending on whether or not necessary care was provided and recorded for individual patients. These binary variables were analysed using an item response model within a multilevel framework using the procedure GLLAMM6 in Stata. Patient scores were obtained for each condition from the rescaled residuals of the item response model and rescaled to range from 0 to 100 . Practice scores were computed for each condition using a multilevel model. These are equivalent to a mean score for each practice adjusted for different pools of patients within practices and the fact that many items were conditional variables.

\section{General Practice Assessment Survey (GPAS) ${ }^{24}$}

The GPAS is a 53 item self-report questionnaire which assesses multiple dimensions of primary care from the perspective of the patient including access, technical care, communication, interpersonal care, trust, knowledge of the patient, nursing care, receptionists, continuity of care, referral, coordination of care, patient recommendation, and overall satisfaction. GPAS measures some constructs with report assessment pairs-for
Box 1 Example of items used in the chronic disease management scores (angina)

- Past 14 months, record of:

- Blood pressure

- Frequency or pattern of angina attacks

- Exercise capacity

- Prescription or advice to take aspirin unless record of contraindication or intolerance

- Prescription of blocker as maintenance treatment if sole therapy

- Action taken on blood pressure if systolic pressure $>160 \mathrm{~mm} \mathrm{Hg}$ or systolic pressure $>140 \mathrm{~mm} \mathrm{Hg}$ and cholesterol $>5.5 \mathrm{mmol} / \mathrm{l}$

- Past 5 years, record of:

- Cholesterol concentration

- Smoking status

- Diet therapy

- Action taken if cholesterol $>5.5 \mathrm{mmol} / \mathrm{l}$

- Weight advice if overweight

- Smoking advice to smokers

- Ever recorded:

- Referral for exercise electrocardiography

- Referral for specialist assessment

The process underlying these criteria has been published elsewhere. ${ }^{22}$

example, "in general, how often do you see your usual doctor?" then "how do you rate this?". Only assessment items are used in the calculation of scale scores and are measured on 6 point scales. Summed scale scores are rescaled to range from 0 to 100. Factor analysis suggests that three dimensions underlie responses to the GPAS: (1) access (includes all the "access" items as well as assessments of "receptionists" and "continuity of care"); (2) patient centredness (includes items from the "communication", "interpersonal", and "knowledge of the patient" scales), and (3) nursing (includes only those items that relate specifically to nursing care) ${ }^{25}$ To limit multiple hypothesis testing, scores were calculated for the two main dimensions of "access" and "patient centredness" based on the sum of three component scales (ranging from 0-300) and used in conjunction with the single item "overall satisfaction" scale (range 0-100).

GPAS has received preliminary validation in the UK. ${ }^{26}{ }^{27}$ Two hundred adult patients were randomly selected from health authority lists for each general practice in the project and sent a copy of GPAS and two reminders (except in one health authority where no reminders were sent).

\section{Analysis of data}

The objectives of the study were to examine the degree to which practice structure predicted climate, and the degree to which structure and climate together accounted for variation in outcomes (fig 1). Multiple regression (using Stata) was used to examine these multivariate relationships. The first regression examined the influence of team structure (independent variables) on team climate (dependent variable). The second group of regressions used team structure and climate as independent variables and the team outcomes as the dependent variables. Because individual responses to the TCI were aggregated at the level of the practice, the number of cases in the multiple regression was far lower than the total number of individual respondents, and both the absolute number of cases and cases per estimated parameter were below that considered optimal. ${ }^{28}$

To examine the relative predictive power of variables, they were all entered into the equation in the first instance and non-significant variables were then removed sequentially (backward selection) using a criterion of $p>0.10$. This more liberal criterion was chosen because of the small sample size. 
Table 1 Practice characteristics $(n=42)$

\begin{tabular}{ll}
\hline Characteristic & \\
\hline Training practice & $31 \%$ \\
Other & $69 \%$ \\
& \\
Practice receiving deprivation payments & $57 \%$ \\
Other & $43 \%$ \\
& \\
Health Authority & $19 \%$ \\
West Penine & $12 \%$ \\
Enfield and Haringey & $19 \%$ \\
Somerset & $17 \%$ \\
South Essex & $19 \%$ \\
Avon & $14 \%$ \\
Bury and Rochdale & \\
& $5910(3650)$ \\
List size* & $14.1(8.5)$ \\
Employed staff* & $5.6(3.6)$ \\
Clinical staff* & $1.3(0.8)$ \\
Practice management staff* & $8.6(5.1)$ \\
Administrative staff* & \\
\hline *Values are mean (SD). & \\
&
\end{tabular}

Because of the relatively small numbers of cases in the analysis, outliers which had a significant influence were identified using Cook's distance (a measure of the change in residuals when a particular case is omitted), removed from the analysis, and the model re-run. A Cook's distance of $8 / \mathrm{N}$ was used to identify outliers rather than the conventional $4 / \mathrm{N}$ because of the small sample size.

Team scores were aggregated at the practice level because they represent the overall team view of effectiveness. Chronic disease management scores were derived from individual patients, but an overall score was computed for each practice. GPAS data were available at the level of the patient. Analyses of GPAS data with panel data techniques and ordinary least squares at the level of the patient, taking into account clustering, were similar to the analysis at the practice level and the practice level analyses are reported.

\section{RESULTS}

\section{Response rate}

Of the 60 practices that took part in the main quality assessment project, 46 (77\%) provided data for the teamwork analysis. The others were not included because of practical reasons (such as lack of resources and a desire to minimise burden in practices recruited late to the project). There were no major differences between participating and non-participating practices in training status or practice size, but practices in receipt of deprivation payments were less likely to participate in the teamwork evaluation.
Questionnaires were sent to all staff employed by the practices $(n=652)$ as well as attached staff such as health visitors and community psychiatric nurses $(n=152)$. However, data on attached staff were variable and analysis was restricted to employed staff. The final response rate was 387/652 (59\%). The response rates from individual practices ranged from $5 \%$ to $100 \%$, with a mean of 65 (SD 26)\%. Practices with a response rate of less than $30 \%(n=4)$ were removed in line with previous teamwork analyses (West, personal communication), leaving 42 practices for analysis. The characteristics of the practices are shown in table 1.

Responses to the GPAS questionnaire in the main quality project were received from 4493 patients, a response rate of $38 \%$. The sample size in the practices included in the teamwork analyses was 3106 .

\section{Skill mix}

Preliminary analysis showed that the skill mix variables SMl and SM2 were highly correlated, and only SMI and SM3 were used in further analyses (Pearson correlation between SMI and SM3 0.13)

\section{Team climate scores}

All scales had satisfactory internal consistency (alpha) and agreement indices $\left(r_{\text {wg(i) }}\right)$, with mean scores on the agreement index ranging from 0.84 to 0.96 and only a small number of practices with scores of $<0.7$ on any scale. TCI subscales were intercorrelated $(r=0.48-0.76)$ and all were highly associated with the overall score, replicating previous work. ${ }^{12}$

\section{Outcome}

The intercorrelations between outcome measures are shown in table 2. All three measures of chronic disease management were highly correlated, as were self-reported team effectiveness and innovation. Patient evaluations were more highly associated with self-reported outcomes than chronic disease management. "Access" and "patient centredness" dimensions of the GPAS were highly correlated with the overall evaluation but only moderately correlated with each other.

\section{Multivariate analyses: structure-process relationships} Two outliers were removed. The final regression model showed that the only predictor of team climate was singlehanded status (regression coefficient $2.38,95 \%$ CI 1.47 to $3.29, \mathrm{n}=40$ ). Team climate was superior in singlehanded practices, and the model explained approximately $41 \%$ of the variance.

\section{Multivariate analyses: structure, process, and outcome relationships}

Longer mean booking interval was associated with higher quality management in all three chronic diseases. Higher TCI scores were associated with higher overall patient evaluations of the practice, higher quality diabetes management, and

\begin{tabular}{|c|c|c|c|c|c|c|c|}
\hline & Innovation & $\begin{array}{l}\text { Asthma } \\
\text { management }\end{array}$ & $\begin{array}{l}\text { Angina } \\
\text { management }\end{array}$ & $\begin{array}{l}\text { Diabetes } \\
\text { management }\end{array}$ & $\begin{array}{l}\text { Overall evaluation } \\
\text { (GPAS) }\end{array}$ & $\begin{array}{l}\text { Access } \\
\text { (GPAS) }\end{array}$ & $\begin{array}{l}\text { Patient centredness } \\
\text { (GPAS) }\end{array}$ \\
\hline $\begin{array}{l}\text { Team } \\
\text { effectiveness }\end{array}$ & $0.48 * *$ & $0.30 *$ & 0.06 & 0.27 & 0.28 & 0.17 & 0.25 \\
\hline Innovation & & 0.19 & 0.04 & 0.27 & $0.36^{*}$ & 0.18 & $0.33^{*}$ \\
\hline $\begin{array}{l}\text { Asthma } \\
\text { management }\end{array}$ & & & $0.53^{* *}$ & $0.69^{* *}$ & 0.10 & -0.09 & 0.17 \\
\hline $\begin{array}{l}\text { Angina } \\
\text { management }\end{array}$ & & & & $0.46^{* *}$ & -0.05 & -0.03 & -0.02 \\
\hline $\begin{array}{l}\text { Diabetes } \\
\text { management }\end{array}$ & & & & & 0.17 & -0.07 & 0.18 \\
\hline $\begin{array}{l}\text { Overall } \\
\text { satisfaction }\end{array}$ & & & & & & $0.77^{* *}$ & $0.73^{* *}$ \\
\hline Access & & & & & & & $0.33^{*}$ \\
\hline
\end{tabular}


Table 3 Regression results: structure, process, and outcome relationships

\begin{tabular}{|c|c|c|c|c|c|}
\hline Outcome variable & Significant predictors & $\begin{array}{l}\text { Regression coefficient } \\
(95 \% \mathrm{CI})\end{array}$ & $p$ value & $\begin{array}{l}\% \text { variance } \\
\text { explained }\end{array}$ & Final $\mathrm{N}$ \\
\hline Overall satisfaction & $\mathrm{TCl}$ & $1.35(0.43$ to 2.26$)$ & 0.005 & $16 \%$ & 42 \\
\hline Access & Singlehanded & 40.2 (25.5 to 54.9 ) & 0.000 & $42 \%$ & 42 \\
\hline Patient centredness & None & NA & NA & NA & 42 \\
\hline Angina management & Booking interval (10 minutes) & $6.71(1.27$ to 12.16$)$ & 0.017 & $11 \%$ & 42 \\
\hline \multirow{2}{*}{ Asthma management } & Booking interval ( 7.5 minutes) & $13.22(0.70$ to 25.74$)$ & 0.039 & $28 \%$ & 42 \\
\hline & Booking interval ( 10 minutes) & 22.16 ( 11.38 to 32.94 ) & 0.000 & & \\
\hline \multirow[t]{3}{*}{ Diabetes management } & $\mathrm{TCl}$ & $2.13(0.20$ to 4.05$)$ & 0.031 & $31 \%$ & 42 \\
\hline & Booking interval ( 10 minutes) & 9.70 (2.79 to 16.63$)$ & 0.007 & & \\
\hline & Number of staff & $0.54(0.12$ to 0.96$)$ & 0.014 & & \\
\hline \multirow[t]{2}{*}{ Innovation } & $\mathrm{TCl}$ & $0.10(0.04$ to 0.17$)$ & 0.003 & $24 \%$ & 40 \\
\hline & Training status & $0.24(0.01$ to 0.46$)$ & 0.038 & & \\
\hline Healthcare team & $\mathrm{TCl}$ & 0.25 (0.17 to 0.34$)$ & 0.000 & $51 \%$ & 40 \\
\hline
\end{tabular}

higher self-reported innovation and effectiveness. Larger team size was associated with better quality diabetes management, training status was associated with self-reported innovation, and singlehanded practices were associated with better access and lower self-reported effectiveness. The percentage of variance explained by the models ranged from $11 \%$ to $51 \%$ (table 3).

\section{DISCUSSION}

The study set out to determine whether practice structure predicts process and whether structure and process predict outcomes (fig 1). Broadly, the data suggest that significant relationships do exist between these variables, which has important implications for developments in primary care which may alter structural organisation and thus influence the climate in primary care teams and eventual outcomes.

\section{Strengths and weaknesses of the study}

Key strengths are the use of multiple, validated, conceptually independent outcome measures, and the involvement of a more representative sample of practices than previous work. However, the direction of causality cannot be determined in an observational study. It is possible that the associations between team climate and outcomes reflect either the effects of outcomes on climate or the influence of a third unmeasured variable.

Power was a fundamental problem. A post hoc power analysis $^{29}$ indicated that regression with 42 cases, six predictors, and $\alpha=0.10$ has reasonable power (85\%) to detect only "large" effects (effect size 0.35); with "medium" effects (effect size 0.15 ) the power is only $49 \%$. The study may therefore be vulnerable to type II errors. Using the more liberal criterion $(p<0.10)$ would also increase the likelihood of type I errors. The results must therefore be seen as preliminary and require replication. The removal of outliers further reduced the sample size. Removal was based on statistical criteria only (for example, to improve model fit), and not on any independent means which has potential for bias.

Conventional regression cannot capture the full relationships in fig 1 where structure affects outcome directly and is also mediated through team process. For example, a large team may have a wider range of clinical skills available, but may not benefit if the climate does not encourage effective sharing of clinical tasks. Examination of these issues would require more complex methods such as simultaneous equation approaches, but these methods have significant sample size requirements.

Clearly, the results require replication in a larger sample, although the difficulties of collecting data from busy primary care teams may make such replication difficult. The aggregation of data at the team level does make such data collection somewhat inefficient: in the present study data from 387 practice staff provided only 42 cases at the level of the team. Furthermore, the exclusion of practices with a response rate of less than $30 \%$ may bias results if response rate itself relates to team climate.

Although a large number of outcome variables was used, these variables are not the only ones that might be influenced by team climate. Team climate might have a stronger relationship with other outcomes which are more obviously influenced by interpersonal and professional functioning-for example, team morale, stress, and professional satisfaction. ${ }^{3}$ The present outcome measures were not chosen on the basis of any explicit theoretical link with team climate.

Finally, the results are dependent on the validity of the outcome measures used. Although the measures of chronic disease management were created using validated consensus group methods, ${ }^{22}$ data on patient evaluations are weakened by low response rate.

\section{Interpretation of the results}

The study found that practice structure predicts process and that structure and process predict outcomes (fig 1).

Of the structural variables entered into the analysis, only singlehanded status was related to a significantly better team climate. The reasons for this are unclear, although team climate was not related to skill mix (which might implicate interdisciplinary issues) or overall staff numbers in the practice (which might suggest an effect of team size). Conflict with GP colleagues has been identified as a stressor for GPs ${ }^{30}$ and a reason for GPs moving to singlehanded status, ${ }^{31}$ and might explain the observed associations. However, doctors working in partnerships also report benefits of teamworking and support. ${ }^{31}$ In addition, team climate scores are based on responses from the entire practice, so relationships among GPs cannot be an entirely satisfactory explanation unless they are a fundamental determinant of climate for all staff.

Length of booking interval was an important predictor of the quality of care in all three chronic conditions, and the advantages of longer consultations have been discussed frequently. ${ }^{32-35}$ Team size was an additional predictor of higher quality diabetes care, which may reflect the positive effects of multidisciplinary working or may be influenced by resource issues as larger practices are better able to find resources and generate the case load needed to support specialist provision-for example, chronic disease management clinics.

The relationship between training status and innovation makes intuitive sense, although the fact that innovation is self-reported means that this association must be treated with caution. Singlehanded status predicted access scores on the GPAS, which also confirms previous work. ${ }^{36}$

The relationships between team climate, innovation, and effectiveness confirm previous research with the $\mathrm{TCI}^{12}$ and are 
vulnerable to the same criticism about common method (selfreport) variance. ${ }^{37}$ The fact that team climate also predicts diabetes management and overall patient evaluation of the practice strengthens the case for its importance.

The mechanisms by which team climate influences chronic disease management remain to be determined. It may involve a non-specific effect whereby climate scores reflect high morale, low stress and professional satisfaction which, in turn, increases staff motivation and effectiveness. This hypothesis is supported by the finding that climate and stress (as measured by the GHQ) are related in primary care teams. ${ }^{37}$ However, the content of the TCI is concerned with specific facets of team climate and more specific mechanisms may be at work. For example, "clarity of objectives" about chronic disease management may be reflected in more effective care provision. It is not immediately clear why team climate impacts on diabetes management and not the other forms of chronic disease management, although this may reflect the fact that diabetes management was most likely to be delivered through specialist clinics at the time of the survey.

The final issue concerns the nature of the link between team climate and patient evaluations. Again, it may be that team climate reflects high morale, and that patients perceive this in terms of the ways in which staff relate to them in face to face encounters (although there was no correlation with GPAS patient centredness). However, other mechanisms cannot be discounted. Team climate may foster innovation and high quality care which, in turn, affects patients' views, although the ability of patients to accurately discern technical aspects of care is unclear. ${ }^{38}$ Of course, an observational study such as this cannot discount the operation of a confounding variable which may explain the observed relationship-for example, the location of the practice and the sociodemographic nature of the practice population-although it should be noted that a crude measure of deprivation (that is, the existence of deprivation payments to the practice) was included in the analysis. It should also be noted that climate predicted patients' overall evaluation of the practice but not the access and patient centredness dimensions. This may reflect, in part, the fact that the overall satisfaction measure relates to the entire practice whereas the patient centredness dimension relates to individual doctors. However, the single item satisfaction scale was highly correlated with the access and patient centredness dimensions.

Determination of the causal mechanisms that underlie these associations remains crucial to increasing conceptual understanding of the basic model shown in fig 1 and allowing the planning of effective team climate interventions. For example, it is important to determine whether singlehanded status is a key determinant of climate or whether the variable represents a proxy for other factors of relevance. ${ }^{39}$ The present analysis used the overall team climate score as the measure of team process, whereas previous analyses ${ }^{12}$ examined individual climate factors. However, it was felt that analysis of multiple process factors would increase the likelihood of type I errors unless corrections were made for multiple testing, and such corrections would be inappropriate given the modest sample size. More precise specification of the causal mechanisms may require additional survey work, although qualitative work (such as interviews with staff and patients from the practices in this analysis, or participant observation) may be equally useful in this regard. As research has indicated that clinical governance initiatives are increasingly focusing on the primary care team as a whole, ${ }^{40}$ an understanding of the influence of climate on team functioning and effective quality of care is of heightened importance.

\section{ACKNOWLEDGEMENTS}

The authors would like to thank the patients and practice staff who took part in the study, Emma Ruff for assistance with administration,

\section{Key messages}

- Current policy highlights the importance of organisationa "culture" in the development and maintenance of quality in healthcare organisations. However, the measurement of "culture" remains problematic.

- One concept that may be relevant to organisational culture is "climate" which represents a team's shared perceptions of organisational policies, practices, and procedures.

- An observational study in primary care examined relationships between practice structure, team climate, and multiple validated outcomes (quality of chronic disease management, patient satisfaction with the practice, and selfreported team outcomes).

- Singlehanded practices had better team climate. Length of booking interval and more positive team climate were associated with better chronic disease management, patient evaluations of the practice, and self-reported innovation and effectiveness.

Martin Roland for assistance with the quality project, Michael West Carol Borrill and Jeremy Dawson (Aston University) for their assistance with the TCI analyses and helpful comments on an earlier draft of the paper, and the referees and editor for additional comments and criticisms. This work was conducted as part of the programme of the National Primary Care Research and Development Centre (NPCRDC) supported by the Department of Health. The views expressed are those of the authors and are not intended to represent the views of NPCRDC or its funders.

\section{Authors' affiliations}

P Bower, S Campbell, B Sibbald, National Primary Care Research and Development Centre, University of Manchester, Manchester M1 3 9PL, UK C Bojke, National Primary Care Research and Development Centre, University of York, York, UK

\section{REFERENCES}

1 Department of Health. Statistical bulletin: statistics for general medical practitioners in England 1987-1997. London: HMSO, 1998.

2 Van Weel C. Teamwork. Lancet 1994;344:1276-9.

3 Firth-Cozens J. Celebrating teamwork. Qual Health Care 1998;7(Suppl):S3-7.

4 Dingwall R. Problems of teamwork in primary care. In: Lonsdale S, Webb A, Briggs T, eds. Teamwork in the personal social services and health care. London: Croom Helm, 1980: 111-37.

5 Beattie A. War and peace among the health tribes. In: Soothill K, Mackay L, Webb C, eds. Interprofessional relations in health care. London: Edward Arnold, 1995: 11-26.

6 Wiles R, Robison J. Teamwork in primary care: the views and experiences of nurses, midwives and health visitors. J Adv Nurs 1994:20:324-30.

7 McClure L. Teamwork, myth or reality: community nurses' experience with general practice attachment. J Epidemiol Community Health 1984;38:68-74.

8 Anderson N, West M. Measuring climate for work group innovation. J Organizational Behav 1998; 19:235-58.

9 Davies H, Nutley S, Mannion R. Organisational culture and quality of health care. Qual Health Care 2000;9:111-9.

10 Shortell S, Rousseau D, Gillies R, et al. Organizational assessment in intensive care units (ICUs): construct development, reliability and validity of the ICU Nurse-Physician questionnaire. Med Care 1991;29:709-26.

11 Poulton B, West M. Primary health care team effectiveness: developing a constituency approach. Health Soc Care 1993;2:77-84.

12 Poulton B. West $M$. The determinants of effectiveness in primary health care teams. J Interprofessional Care 1999:13:7-18.

13 Goni S. An analysis of the effectiveness of Spanish primary health care teams. Health Policy 1999;48:107-17

14 Peiro J, Gonzalez-Roma V, Ramos J. The influence of work-team climate on role stress, tension, satisfaction and leadership perceptions. Eur Rev Appl Psychol 1992:42:49-56

15 Kivimaki $M$, Sutinen R, Elovainio $M$, et al. Sickness absence in hospital physicians: 2 year follow up study on determinants. Occup Environ Med 2001;58:361-6.

16 Campbell S, Hann M, Hacker J, et al. Identifying predictors of high quality care in English general practice: observational study. BM 2001;323:784-7.

17 Kozlowski S, Hattrup K. A disagreement about agreement: disentangling issues of consistency versus consensus. J Appl Psychol 1992;77:161-7.

18 James L, Demaree R, Wolf $G$. Estimating within-group interrater reliability with and without response bias. J Appl Psychol 1984;69:85-98 
19 James L, Demaree R, Wolf $G$. $r_{\text {wg }}$ an assessment of within-group interrater agreement. J Appl Psychol 1993;78:306-9.

20 Schmidt F, Hunter J. Interrater reliability co-efficients cannot be computed when only one stimulus is rated. J Appl Psychol 1989;74:368-70.

21 Poulton B, West M. Effective multidisciplinary teamwork in primary health care. J Adv Nurs 1993;18:918-25.

22 Campbell S, Roland M, Shekelle P, et al. The development of review criteria for assessing the quality of management of stable angina, adult asthma and non-insulin dependent diabetes mellitus in general practice. Qual Health Care 1999:8:6-15.

23 Campbell S, Hann M, Hacker J, et al. Quality assessment for three common conditions in primary care: validity and reliability of review criteria developed by expert panels for angina, asthma and type 2 diabetes. Qual Saf Health Care 2002;11:125-30.

24 Roland M, Holden J, Campbell S. Quality assessment for general practice: supporting clinical governance for primary care groups. Manchester: NPCRDC, 1998

25 Bower P, Mead N, Roland M. What dimensions underlie patient responses to the General Practice Assessment Survey? A factor analytic study. Fam Pract 2002;19: 489-95.

26 Ramsay J, Campbell J, Schroter S, et al. The General Practice Assessment Survey (GPAS): tests of data quality and measurement properties. Fam Pract 2000;1 17:372-9.

27 Campbell J, Ramsay J, Green J. Age, gender, socioeconomic, and ethnic differences in patients' assessments of primary health care. Qual Health Care $2001 ; 10: 90-5$

28 Ullman J. Structural equation modelling. In: Tabachnick B, Fidell L, eds. Using multivariate statistics. New York: Harper Collins, 1989: 709-81 1

29 Erdfelder E, Faul F, Buchner A. GPOWER: a general power analysis program. Behav Res Methods Instrum Comput 1996;28:1-11.

30 Firth-Cozens J. Individual and organizational predictors of depression in general practitioners. Br J Gen Pract 1998;48:1647-51.
31 Green JM. The views of singlehanded general practitioners: a qualitative study. BM 1993;307:607-10.

32 Freeman G, Horder J, Howie J, et al. Evolving general practice consultation in Britain: issues of length and context. BM 2002;324:880-2.

33 Howie J, Porter A, Forbes J. Quality and the use of time in general practice: widening the discussion. BM 1989:298:1008-10.

34 Howie JG, Heaney DJ, Maxwell M, et al. Developing a 'consultation quality index' (CQl) for use in general practice. Fam Pract 2000;17:455-61.

35 Wilson A, Childs S. Systematic review of consultation length in general practice: a report to the Scientific Foundation Board of the RCGP. Leicester: University of Leicester, 2001.

36 Campbell J, Ramsay J, Green J. Practice size: impact on consultation length, workload, and patient assessment of care. Br J Gen Pract 2001;51:644-50.

37 Carter A, West M. Sharing the burden: teamwork in health care settings In: Firth-Cozens J, Payne R, eds. Stress in health professionals: psychological and organisational causes and interventions. Chichester: John Wiley and Sons, 1999: 191-202.

38 Chapple A, Campbell S, Rogers A, et al. Users' understanding of medical knowledge in general practice. Soc Sci Med 2001;54:121524

39 Baines D. A healthy disposition? The use and limitations of the characteristics approach to general practice research. Br J Gen Pract 2001;51:749-52.

40 Campbell S, Sheaff R, Sibbald B, et al. Implementing clinical governance in English primary care groups/trusts: reconciling quality improvement and quality assurance. Qual Saf Health Care $2002 ; 11: 9-14$

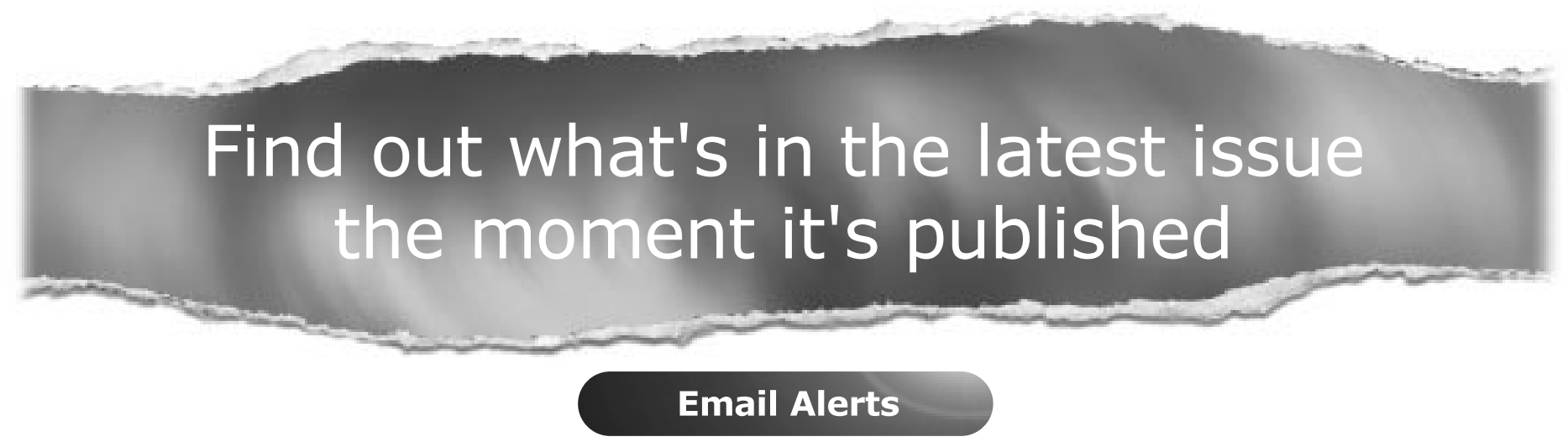

Sign up to receive the table of contents by email. You can select different alerts including: Table of Contents (full), and Quality and Safety in Health Care related announcements.

\section{www.qshc.com}

